\title{
Using Learning Analytics and Visualization Techniques to Evaluate the Structure of Higher Education Curricula
}

\author{
Artur Mesquita Barbosa ${ }^{1}$, Antonio Nilo de Araujo Neto ${ }^{1}$, \\ Emanuele Santos ${ }^{1}$, João Paulo P. Gomes ${ }^{1}$ \\ ${ }^{1}$ Departamento de Computação \\ Universidade Federal do Ceará (UFC) - Fortaleza - CE - Brazil \\ \{artur, nilo, emanuele, jpaulo\}@lia.ufc.br
}

\begin{abstract}
In this paper, we propose a data mining technique that evaluates a curriculum's structure based on academic data collected from Computer Science students from 2005 to 2016. Our approach is based on the Synthetic Control Method (SCM), which builds a linear model describing the relation between courses based on student performance information. The proposed model is compared to a linear regression model with positive coefficients. In addition to providing the relation between courses, it can also be used to predict students' grades in a specific course based on their previous grades. The results are visualized in a user-friendly tool, which allows for contrast and comparison between the official structure and the structure found based on the data.
\end{abstract}

\section{Introduction}

One of the most difficult challenges that educators and leaders in higher education face today is reducing the high student dropout rates in their institutions. In recent years, there has been an increase in the number of approaches that apply Learning Analytics techniques trying to solve this problem [Anuradha and Velmurugan 2015, Kantorski et al. 2016, Badr et al. 2014, de Brito et al. 2014]. These approaches use academic and socioeconomic data collected from students to early diagnose the students prone to evasion and then take appropriate measures for preventing it.

However, the structure of the curriculum also plays a big role in the development of students' knowledge and their performance [Anuradha and Velmurugan 2015]. Despite this fact, research using learning analytics to analyze curricula, pointing out strengths and weaknesses, are not very common in the literature. Additionally, there is also a significant demand for tools to assist educators in using data and evidence to build better curricula.

In this paper, we propose a data mining technique that evaluates a curriculum's structure based on academic data collected from undergraduate students. Our approach is based on the Synthetic Control Method (SCM) which has been successfully applied in previous studies in social science and economics [Hinrichs 2012, Abadie et al. 2010]. The SCM builds a linear model describing the relation between courses based on a dataset of student performance information. In the resulting linear model, each course is described as a convex combination of previous courses, thus improving the interpretability of the model. In addition to providing the relation between courses, the proposed method can also be used to predict students' grades in a specific course based on their previous grades. The results can be visualized inside a user-friendly tool. 


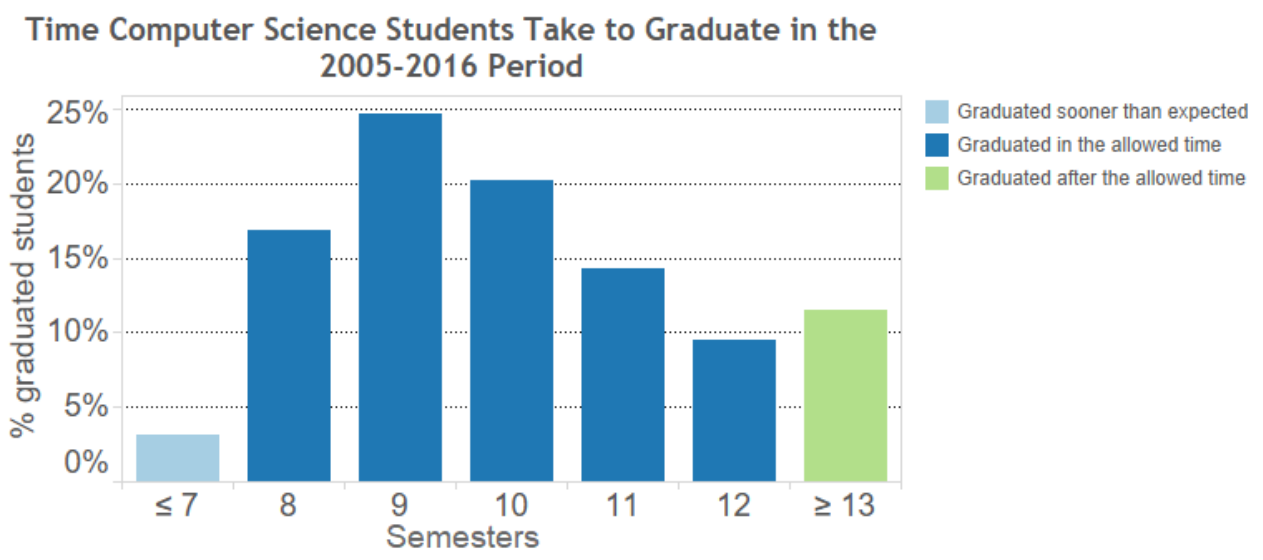

Figure 1. Distribution of graduation time in semesters for Computer Science students. The observed period is from 2005 to 2016. Most of the students take more time than the standard time suggested to graduate ( 8 semesters).

We verified the feasibility of our approach by applying it to a dataset collected from more than 800 Computer Science students enrolled in the same curriculum along a 10-year period. We compared our results to a linear regression model with positive coefficients. Our tests showed that our approach produces more interpretable results that led to several important insights regarding the structure of the computer science program in analysis. We also use the same dataset to describe how our proposed visualization tool can be used by educators to analyze the results and to compare with the official structure of the curriculum and find hidden relations between courses.

The remainder of this paper is organized as follows. In Section 2, we explain the collected dataset and the SCM Method. In Section 3, we describe our experiments and our results. In Section 4, we provide a short description of the developed visualization tool before discussing our results in more detail in Section 5. In Section 6, we review the related work and finally, in Section 7, we conclude our paper and outline directions for future work.

\section{Methodology}

In this section we describe the learning model used to evaluate the structure of a curriculum. We start by describing the dataset used in our experiments and then, we present the Synthetic Control Method (SCM) and explain how it is used in our analysis. Roughly speaking, SCM builds a linear model that tries to predict the grades of students in a given course based on their grades in previous courses. The coefficients of the linear model reveal the courses that are used to predict the grade. Using this result, we can infer a possible dependency relation between courses.

\subsection{Dataset description}

We acquired a dataset of 892 Computer Science students enrolled from 2005 to 2016 in a curriculum that was in place since the first semester of 2000. This program admitted students in this curriculum until 2015, and then, in 2016, a new curriculum was established. The students were followed until 2016. Despite being a high-quality Computer Science program, it also suffers from evasion and retention. From 2005 to 2015, the average dropout rate was superior to $45 \%$. Also, most of the students take longer to graduate 
Table 1. Description of the attributes collected for each mandatory course taken by the Computer Science students in the dataset.

\begin{tabular}{lll}
\hline Attribute & Type & Description \\
\hline Course ID & int & A unique number identifying the course. \\
Grade & float & Final grade for the course. \\
Attendance & float & Percentage of attendance (0 to 100) for the course. \\
Semester & float & Semester when the course was taken. \\
GPA & float & $\begin{array}{l}\text { Partial GPA of the most recent semester before the course was taken, 0 if it was taken in the first semester } \\
\text { (GPA is the weighted grade point average, where the weights are the courses' credits). }\end{array}$ \\
\hline
\end{tabular}

than the suggested 8-semester time: the average time is 9 semesters, and as we can see in Figure 1, there is a large concentration in 9 to 12 semesters. These numbers may indicate that the structure of the curriculum could be playing a part on this behavior and so we would like to know the answer for the following question: is there any relationship between the courses' grades and their prerequisites?

In order to graduate, the students in this curriculum are required to take 31 mandatory courses (they must also take a certain number of elective courses, but these were not considered in this work). The provided dataset contained information about the mandatory courses taken by each student and it is described in Table 1 .

Notice that for the students still active or the students that dropped out, we have the information only for the courses taken until then. Besides, there were a few cases when the student was able to take a course without fulfilling its prerequisites (this is allowed in some specific situations by the program). When this occurred, we left that course out of the analysis.

\subsection{Synthetic Control Method}

The Synthetic Control Method (SCM) was first introduced in the seminal paper [Abadie and Gardeazabal 2003]. In order to try to estimate the impact of terrorism in the Basque Country's economy in the 70's, the authors used regions in Spain to find a counterfactual group to represent a synthetic Basque Country, where the terrorism has never happened and, in this way, they would measure the differences between the real Basque Country and its synthetic counterpart. To do so, the work finds a convex combination of the Spanish regions that best represent the data describing the Basque Country in the pre-terrorism period.

This approach could also be used to infer the impact of a specific measure in a undergraduate course, like changing its instructor, or changing the methods used in the classroom. In our work, however, we are interested in the convex combination produced by the method, from which we can extract some information about the relations between different courses in the curriculum.

We now describe the method used in this work. We first assume we have a matrix $Y_{n \times m}$, where each row represents the grades of a student, and each column represents the grades obtained by all the students in a given course. For now, we assume there is no missing data. Also, let $X_{k \times m}$ be a predictor matrix, which contains $k$ different measures regarding each of the courses. The choice of which measures to use is at the researcher's discretion. In this paper, we selected the following measures as predictors: average grade 
of students with final grade above 7 (hereby named as A grades, or B otherwise) in that course, average grade of B graded students, average grades overall, average attendance of A graded students, average attendance of B graded students, average attendance overall, average GPA from A graded students at the moment they enroll in that course, average GPA from B graded students, average number of A graded students in that course, average number of B graded students.

Our goal is to find a combination of course grades that best represent a certain target course. Consider that the last column in the $X$ and $Y$ matrices, denoted by $X_{1}$ and $Y_{1}$, contain the data related to our target unit (treatment unit, in Abadie and Gardeazabal's original work). The rest of the matrices, $X_{0}$ and $Y_{0}$, comprehend data from the rest of the courses, hereby named control units.

Let $W_{m-1 \times 1}$ be a vector where $0 \leq w_{i} \leq 1$ and $\sum_{0}^{m-1} w_{i}=1$. Our purpose is thereby to find such a vector, where the synthetic group, $Y_{0} W$, best represents the target unit output $Y_{1}$. To measure this representation, we use a $V_{k \times k}$ diagonal matrix in which each element $v_{i, i}$ accounts for the relative importance of the $i$-th predictor when comparing the difference between the synthetic group and the target unit. We arrive at an iterative optimization loop of the form:

$$
\begin{gathered}
V=\underset{V}{\arg \min }\left(\left|Y_{1}-Y_{0} W(V)\right|\right) \\
W(V)=\underset{W}{\arg \min }\left(\left(X_{1}-X_{0} W\right)^{T} V\left(X_{1}-X_{0} W\right)\right)
\end{gathered}
$$

To obtain the desired output, we apply this method considering one course as the target unit at a time. In addition, we only use courses as control units for a certain target if they occur before that target in the curriculum; i. e. for a target unit in the $M$-th semester, $M>1$, only the courses in semester $M-1$ or earlier are used as controls.

\section{Experiments and results}

The first part of the experiments consists in using only grades from students that eventually graduated the program. This way, we maintain the number of samples for each target unit constant, avoiding missing data. We then compare the SCM's results with those obtained from linear regression with positive weights. We decided to use constrained linear regression (positive weights) because negative relations between grades of different courses do not seem to make sense.

To quantify the performance of each method we computed the error between the predicted and the real grades. This result was averaged for all courses and all students of the dataset. The results for both methods can be seen in Table 2. Both models had a good performance in the grade prediction task. There is no course with an error greater than $19 \%$ and both methods achieved an average error of $13 \%$. It is important to mention that this is a remarkable result for SCM, since the linear regression is designed to minimize the error between the model and the real grades, while SCM is designed not only to accurately predict the grades but also to provide models with limited non-zero coefficients.

We may also verify how the coefficients relate to the course's prerequisites. For that, we consider the prerequisite prediction task as a binary classification problem. In this 
VI Congresso Brasileiro de Informática na Educação (CBIE 2017)

Anais do XXVIII Simpósio Brasileiro de Informática na Educação (SBIE 2017)

Table 2. Relative mean errors of each course for graduated students.

\begin{tabular}{lcc||lcc}
\hline \hline Course & SCM & Constr. Regression & Course & SCM & Constr. Regression \\
\hline Calculus II & 0.16 & 0.15 & Databases I & 0.11 & 0.08 \\
Physics I & 0.15 & 0.15 & ProgLang I & 0.17 & 0.12 \\
DataStruct & 0.16 & 0.14 & NumMeth II & 0.11 & 0.12 \\
Prog II & 0.13 & 0.14 & Networks & 0.12 & 0.11 \\
DataTransf & 0.13 & 0.11 & Databases II & 0.09 & 0.10 \\
Logic & 0.16 & 0.13 & SoftEng & 0.09 & 0.12 \\
ProbStat & 0.16 & 0.13 & AI & 0.16 & 0.17 \\
GraphAlgo & 0.16 & 0.16 & OS I & 0.11 & 0.14 \\
Prog III & 0.10 & 0.12 & SysProject & 0.08 & 0.09 \\
CompArch I & 0.10 & 0.11 & Automata & 0.14 & 0.16 \\
Algorithms & 0.15 & 0.15 & Compilers & 0.11 & 0.18 \\
NumMeth I & 0.14 & 0.14 & CompTheory & 0.13 & 0.16 \\
CG I & 0.19 & 0.12 & & & \\
\hline \hline
\end{tabular}

Table 3. Average Error and F1 Score for SCM and Linear Regression.

\begin{tabular}{lc|c}
\hline & Synthetic Control Method & Linear Constrained Regression \\
\hline Average Error & 0.13 & 0.13 \\
F1 Score & 0.27 & 0.20
\end{tabular}

setup, each possible required course is classified as being part of the prerequisites or not being part of the prerequisites. By using this framework, we can assess the performance of the methods using the standard F1 score. For both SCM and linear regression, we considered a course as a prerequisite if its associated coefficient is greater than 0.1 . In Table 3, we present the average results for the error and the F1 score metrics.

The results in Table 3 show that SCM achieved a F1 score greater than the linear regression's. That performance indicates that SCM's predicted dependency between courses is closer to the official curriculum. It is also important to notice that both methods had low F1 scores (F1 scores range from 0 to 1 ). This fact is expected because we considered a simplified scenario in which we wish to infer the dependency of courses only using the performance of the students. Although using this simplified assumption, several interesting insights are generated by our method. In the next section, we describe our developed visualization tool and then, in Section 5, we explain our insights and how we used this tool to find them.

\section{Visualization tool}

Sometimes evaluating the program's curriculum by only looking at numbers can be very difficult for educators who do not have the necessary knowledge in statistics and machine learning. To account for that, we developed a user-friendly interactive web visualization tool that provides all the information educators need to perform an intuitive and in-depth analysis. 
VI Congresso Brasileiro de Informática na Educação (CBIE 2017)

Anais do XXVIII Simpósio Brasileiro de Informática na Educação (SBIE 2017)

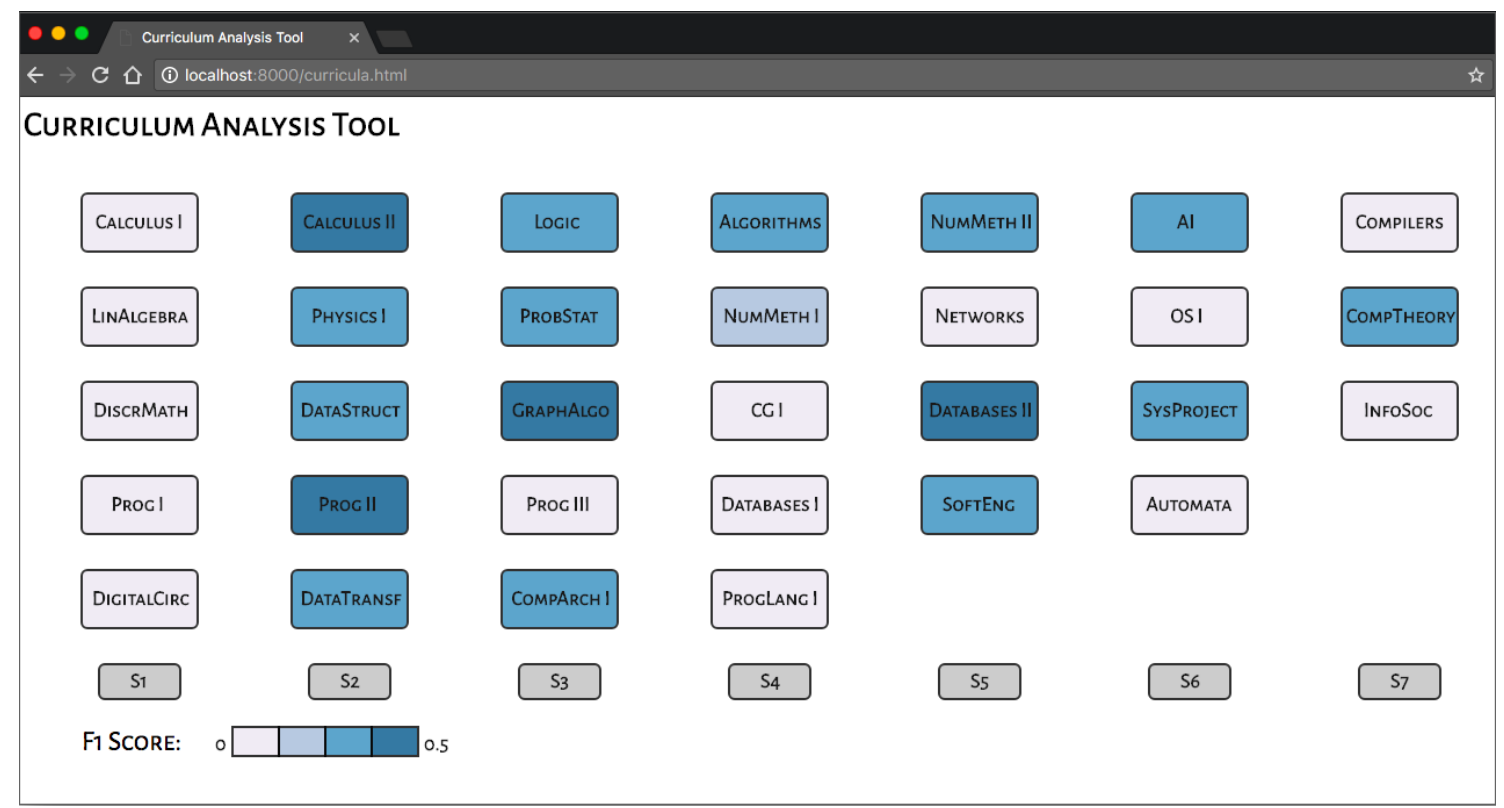

Figure 2. Visualization tool's overview. The curriculum's mandatory courses are displayed in chronological order. Each course is colored in shades of blue (the saturation increases according to its F1 score).

The visualization tool is composed of three main views: the overview, the course influence view and the influence value view. When the analysts load the tool on the browser, the first view they see is the overview (see Figure 2). In this view, the curriculum's mandatory courses are displayed in chronological order, each semester is represented by a column and each course is depicted by a box labeled with its code or a nickname provided by the analyst (the full name of the course is shown as a tooltip when passing the mouse over it). Also, each course is colored in shades of blue, and the darker the shade is, the larger is the course's F1 score. The scale of these values is shown in the legend at the bottom-left of the page. The analyst can also click on the legend's boxes to create a threshold for this value and filter only the courses that satisfy the selected threshold. The main purpose of this view is to show the differences between the official curriculum's structure and the structured acquired from the data at a single glance and without displaying too much detail [Munzner 2014, Chapter 5]. In the first versions of this tool, we also considered showing the courses' prerequisites by connecting them with edges but we soon decided not doing so to avoid clutter.

After looking at the overview, the analyst can investigate each course more deeply using the course influence view (see Figure 3). To open this view, the user clicks on the course's box, the view focuses only on the analysis results of that course. In this view, the selected course is shown in gray, its required courses that were classified as influent are colored in a dark blue, its required courses that were not classified as influent are colored in yellow, and the courses that are not required but were classified as influent are colored as a light blue. If the analysts want to go even deeper and see how much influence each course receives, they can also click on the "Check influence value" button, and bring up the course's influence value view (see Figure 4). The visualization changes, fading out the yellow-colored boxes and changing the colormap to a shade of red now encoding 


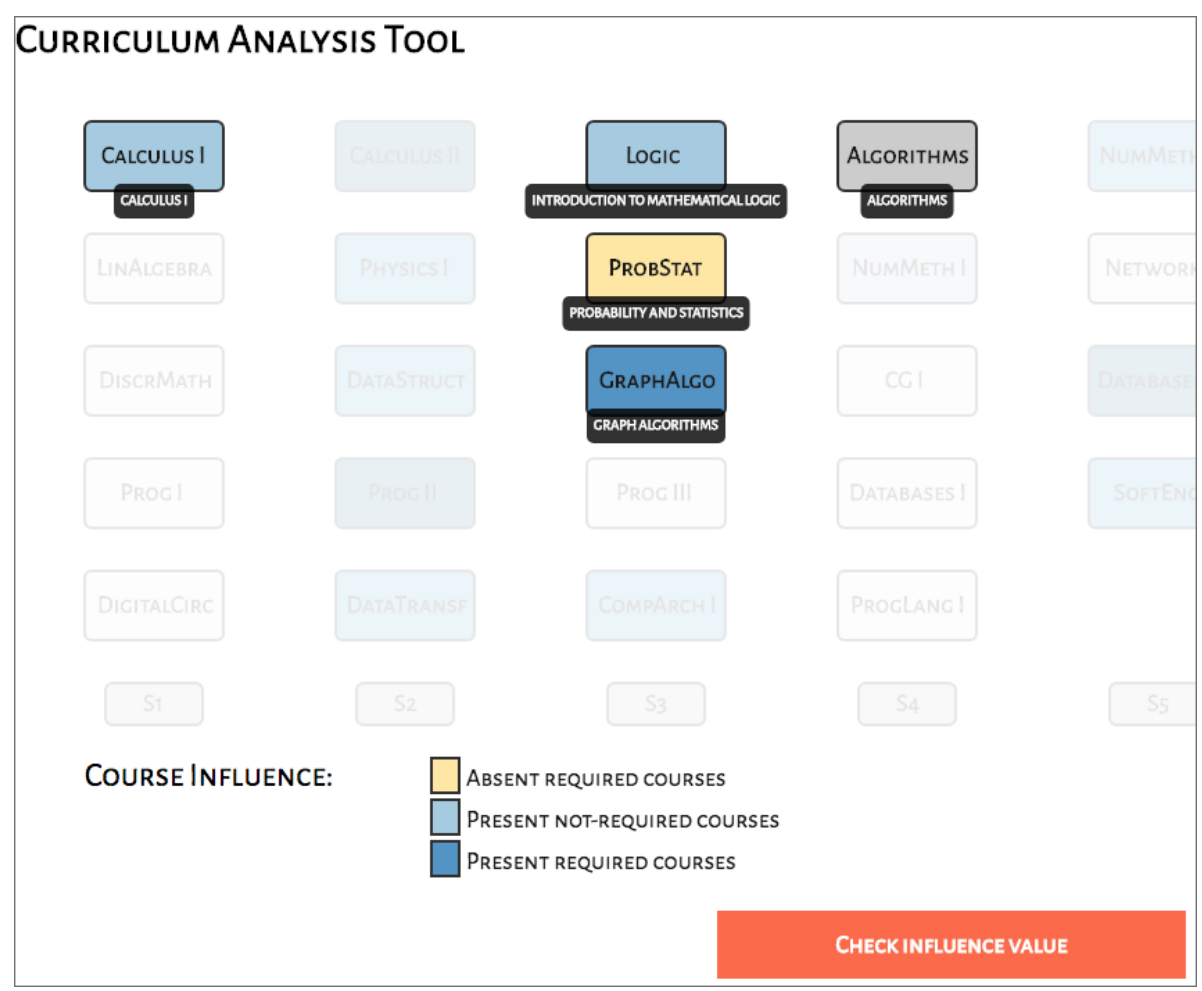

Figure 3. Course influence view. The courses that influence the selected Algorithms shown in gray are displayed in shades of blue (all the other courses are faded out). The prerequisite the model considered to influence Algorithms is shown in dark blue, and the real prerequisite considered not to influence it shown in yellow.

the influence rate. In this colormap, the saturation increases according to the influence rate. Similarly as the legend boxes in the overview, the legend boxes in this view also allow filtering courses based on a threshold value. The user can still perceive the official required courses because they have a thicker border around their boxes (see Databases I in Figure 4). By clicking anywhere on the background, the users are brought back to the overview.

This tool allows the users to see how different the official curriculum is from the model built from the data, exploring different levels of detail. In the next section, we describe how we used this tool to gain some insights about the SCM results.

\section{Discussion}

One of the main questions we wanted to answer about the analysis was why we obtained a low value for the F1 score. By using the visualization tool, starting at the overview (see Figure 2), we can see that, in fact, the official curriculum did not match well with the model because of the high number of light and medium blue boxes. A plausible explanation for this is that our method uses information about only the grades of the students, and this is not enough to infer the relations between courses. In fact, other aspects such as methodologies, professors, the amount of theory versus practice are not being considered in our work and may have a significant impact on the results. However, there were some interesting findings. We can see in Figure 2 that only a small number 


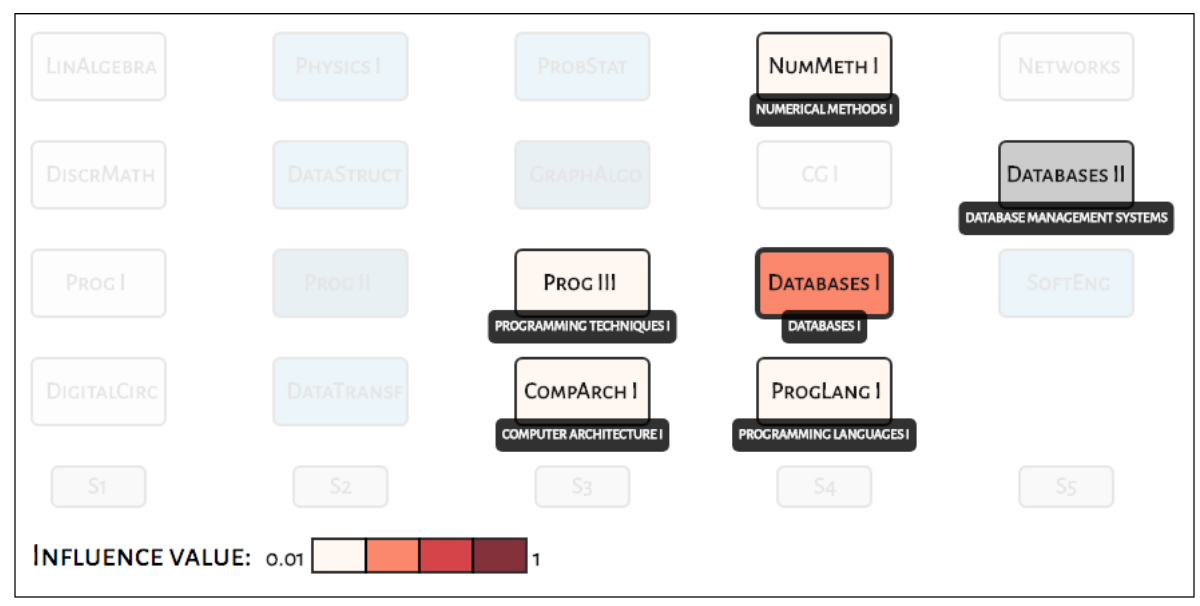

Figure 4. Influence value view for Databases II, in which the saturation increases according to the influence rate. In the model. Databases II is more influenced by Databases $I$, being consistent with the official curriculum.

of courses have the darkest shades of blue, which means that few courses in the official curriculum matched well with the model. For example, Databases II in semester 5, has only Databases $I$ as a prerequisite in the official curriculum. If we see the influence value view of Databases II, as shown in Figure 4, we can conclude that although other courses were considered to influence Databases II, the model assigned a higher value to Databases I.

Going back to the overview in Figure 2, we notice that most of the courses fit an intermediary value for the F1 score. In general, this is when the model does not include some of the prerequisites present in the official curriculum. In the case of Algorithms, for example, the model did not include the prerequisite ProbStat (see Figure 3). However, this case was very interesting because in a recent update of the curriculum in 2016, this prerequisite was removed from the list of Algorithms' prerequisites and so, in the end, the model agreed with reality.

Another interesting case is when the model considered other courses to influence a certain course, outside the list of its prerequisites, also causing a low F1 Score. Take for example, $A I$ (Artificial Intelligence) in Figure 5. In this case, the model included DiscrMath and DataStruct in the courses that influence AI, but that are not direct prerequisites. However, by inspecting other courses' prerequisites we will discover that they are indeed indirect prerequisites and the student must have attended those courses before taking $A I$.

\section{Related Work}

Most of the previous works using Learning Analytics to detect the students' performance [Ogunde and Ajibade 2014, Badr et al. 2014, Baradwaj 2011, de Brito et al. 2014] classified the students using algorithms more suitable for interpretation, such as decision trees or rule induction, mainly because their goal was to identify the reasons for the students' behaviors and these works were limited to the standard visualization tools provided in the machine toolbox they used, when available. Our approach used SCM and to help its interpretability, we developed a specific visualization tool. Other works focused on the development of curriculum mining tools and 


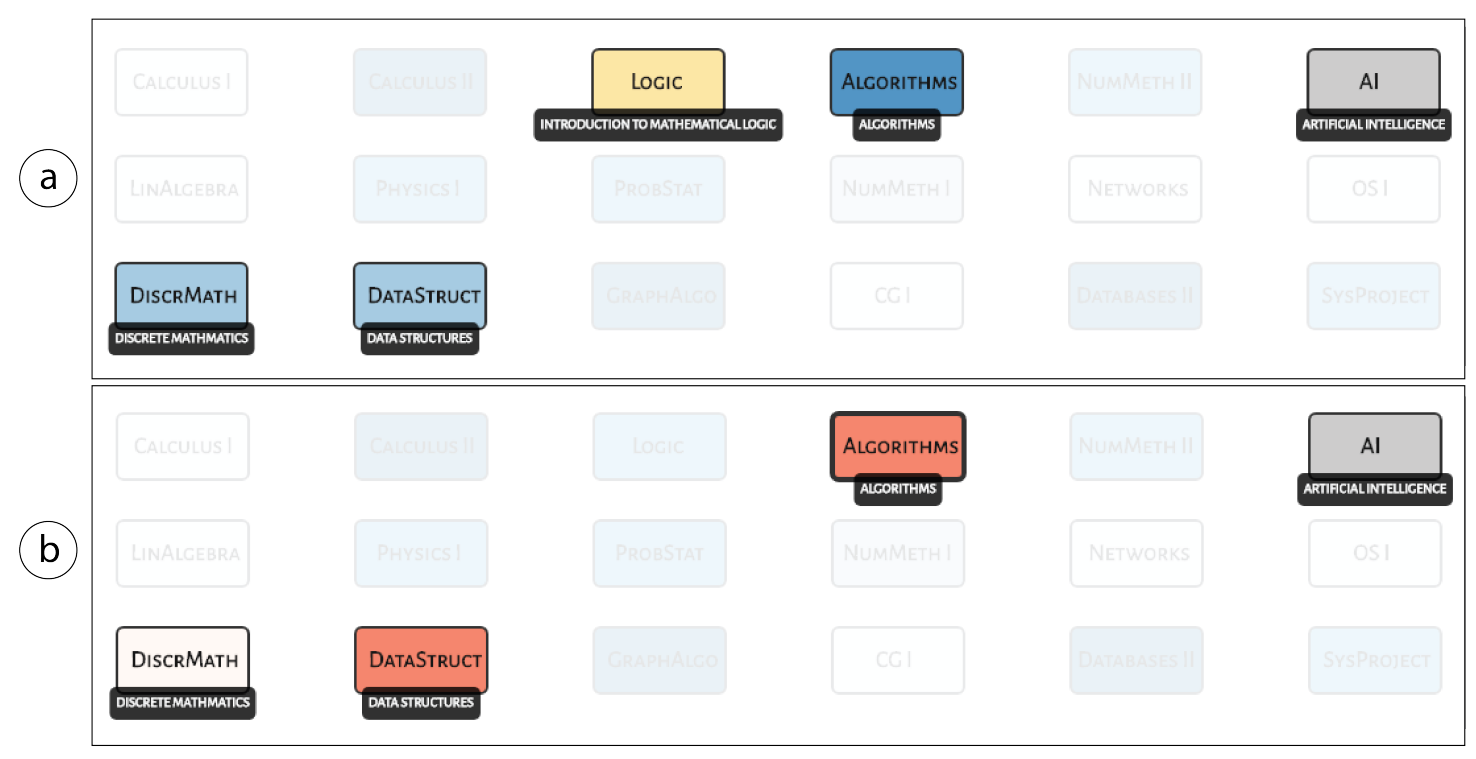

Figure 5. Inspecting the courses influencing Al: a) Course influence view and b) Influence value view for $\boldsymbol{A l}$.

techniques [Pechenizkiy et al. 2012, Wang and Zaïane 2015] with the goal of finding frequent paths. Another work also provided recommendations to the students' career in the program [Campagni et al. 2015]. Our work focused on finding the relationships between courses, grades and prerequisites. Finally, another work provided an interactive visualization of academic trajectory patterns using nodes representing courses connected by edges to show students' path [Jordão et al. 2014]. The thicker the edges, the more students follow the same path. However, there is no Machine Learning algorithm being applied. Our work integrates Machine Learning and Visualization.

\section{Conclusions}

In this paper, we presented a data mining technique that evaluates a curriculum's structure based on academic data collected from 892 Computer Science students from 2005 to 2016. Our approach was based on the Synthetic Control Method (SCM), which builds a linear model describing the relation between courses based only on student performance information. We compared the proposed model to a linear regression model with positive coefficients, achieving better results. In addition to providing the relation between courses, our approach can also be used to predict students' grades in a specific course based on their previous grades. We also developed a Web based tool to visualize the results. This tool allows for contrast and comparison between the official curriculum and the model built based on the data and can be used by educators to evaluate the current structure of the curriculum, find hidden relations between courses and build hypotheses about the model. As a future work, we would like to use the data to build customized curricula, suggesting the best order for taking the courses, respecting the curriculum's constraints, and that would improve students' performance. This would require devising a more complex visualization tool. We are also considering ways to incorporate other information about the courses and how to encode this information to try to improve our F1 scores. 
VI Congresso Brasileiro de Informática na Educação (CBIE 2017)

Anais do XXVIII Simpósio Brasileiro de Informática na Educação (SBIE 2017)

\section{References}

Abadie, A., Diamond, A., and Hainmueller, J. (2010). Synthetic control methods for comparative case studies: Estimating the effect of california's tobacco control program. Journal of the American Statistical Association, 105(490):493-505.

Abadie, A. and Gardeazabal, J. (2003). The economic costs of conflict: A case study of the basque country. American Economic Review 93. 113-132.

Anuradha, C. and Velmurugan, T. (2015). A data mining based survey on student performance evaluation system. 5th IEEE International Conference on Computational Intelligence and Computing Research, IEEE ICCIC 2014, (December 2014):43-47.

Badr, A., Din, E., and Elaraby, I. S. (2014). Data Mining: A prediction for Student's Performance Using Classification Method. World Journal of Computer Application and Technology, 2(2):43-47.

Baradwaj, B. K. (2011). Mining Educational Data to Analyze Students' Performance. IJACSA) International Journal of Advanced Computer Science and Applications, 2(6).

Campagni, R., Merlini, D., Sprugnoli, R., and Verri, M. C. (2015). Data mining models for student careers. Expert Systems with Applications, 42(13):5508-5521.

de Brito, D. M., Júnior, I. A. d. A., Queiroga, E. V., and do Rêgo, T. G. (2014). Predição de desempenho de alunos do primeiro período baseado nas notas de ingresso utilizando métodos de aprendizagem de máquina. Anais do Simpósio Brasileiro de Informática na Educação, 25(1):882-890.

Hinrichs, P. (2012). The effects of affirmative action bans on college enrollment, educational attainment, and the demographic composition of universities. The Review of Economics and Statistics, 94(3):712-722.

Jordão, V., Gama, S., and Gonçalves, D. (2014). EduVis: Visualizing educational information. 8th Nordic Conference on Human-Computer Interaction, NordiCHI 2014, pages 1011-1014.

Kantorski, G., Flores, E. G., Schmitt, J., Hoffmann, I., and Barbosa, F. (2016). Predição da Evasão em Cursos de Graduação em Instituições Públicas. Brazilian Symposium on Computers in Education (Simpósio Brasileiro de Informática na Educação - SBIE), 27(1):906.

Munzner, T. (2014). Visualization Analysis \& Design. A K Peters Visualization Series. CRC Press - Taylor \& Francis Group, Boca Raton, FL, 1 edition.

Ogunde and Ajibade (2014). A Data Mining System for Predicting University Students' Graduation Grades Using ID3 Decision Tree Algorithm Ogunde A. O 1 . and Ajibade D. A 1 . Computer Science and Information Technology, 2(1):21-46.

Pechenizkiy, M., Trcka, N., De Bra, P., and Toledo, P. (2012). CurriM: Curriculum Mining. Proceedings of the 5th International Conference on Educational Data Mining, (i): $1-4$.

Wang, R. and Zaïane, O. R. (2015). Discovering Process in Curriculum Data to Provide Recommendation. In Proceedings of the 8th International Conference on Educational Data Mining, pages 580-581. 